\title{
Competition among the Axonal Projections of an Identified Neuron Contributes to the Retraction of Some of Those Projections
}

\author{
Wen-Biao Gan and Eduardo R. Macagno \\ Department of Biological Sciences, Columbia University, New York, New York 10027
}

\begin{abstract}
AP neurons in the embryonic leech CNS extend lateral projections to peripheral targets through the ganglionic nerve roots and longitudinal projections toward neighboring ganglia through the connective nerves. The lateral projections grow extensively in the periphery; in contrast, the longitudinal projections achieve relatively little growth and eventually retract, the majority having essentially disappeared by the end of embryogenesis. Cutting both nerve roots, which eliminates both lateral projections, however, induces the longitudinal projections of the AP neuron to begin to grow rapidly toward adjacent ganglia within $14 \mathrm{hr}$ after the axotomy. By using a laser microbeam to cut just the lateral projections of the AP cells, we further show that it is indeed the loss of its lateral projections, and not a secondary response to the cutting of other components of the root nerves, that induces the longitudinal projections of the AP cell to grow extensively. In addition, we report
\end{abstract}

that reducing the outgrowth of the lateral projections by: (1) cutting only one lateral projection, or (2) ablating pioneer neurons required by the AP neuron to establish its peripheral arbor, also results in a significant increase in the growth of the longitudinal projections. Finally, we demonstrate that increasing the outgrowth of the longitudinal projections by ablating the AP cells in adjacent ganglia results in a significant reduction in the outgrowth of the lateral projections. Taken together, these results indicate, first, that the longitudinal and lateral projections usually grow at the expense of each other, and second, that normally the extensive outgrowth of its lateral projections is a necessary condition for a developing AP neuron to retract its longitudinal projections.

Key words: axonal retraction; axonal outgrowth; cell-cell interactions; axotomy; competition; development; leech
In establishing their particular branching patterns, neurons often generate some neurites that are later retracted (Land and Lund, 1979; Innocenti, 1981; O'Leary et al., 1981; McLoon, 1982; Wallace, 1984; Stanfield and O'Leary, 1985; Glover and Mason, 1986; Gao and Macagno, 1987a,b; Jellies et al., 1987; Baptista and Macagno, 1988; Callaway and Katz, 1990; O'Rourke et al., 1994). Synapse elimination at the neuromuscular junction (Redfern, 1970; Balice-Gordon and Lichtman, 1993) and the segregation of LGN projections to the visual cortex in the formation of ocular dominance column (LeVay et al., 1978, 1980) are well known examples of this phenomenon. It has been suggested that axonal retraction may play an important role in creating connectional diversity and specificity in developing nervous systems (O'Leary, 1992). The mechanisms underlying axonal retraction in the developing nervous system, however, are not well understood.

At the neuromuscular junction, one of the most extensively studied systems in which process pruning occurs, some of the axonal terminals of each motoneuron disappear during a period of transition from multiple innervation to single innervation (for review, see Colman and Lichtman, 1993). This transition is thought to occur as a result of a local competition among the

Received Nov. 27, 1996; revised March 13, 1997; accepted March 21, 1997.

This work was supported by grants from the National Science Foundation and National Institutes of Health. We thank Drs. Laura Wolszon, Beatrice Passani, Wei-Qiang Gao, and Fei-Chi Zhuong for their help and useful discussions. We also thank Nicholas Necles for assistance with photographic work.

Correspondence should be addressed to Dr. Eduardo R. Macagno, Department of Biological Sciences, 1003 Fairchild Building, Columbia University, New York, NY 10027.

Dr. Gan's present address: Department of Anatomy and Neurobiology, Washington University School of Medicine, P. O. Box 8108, 660 South Euclid Avenue, St. Louis, MO 63110

Copyright (C) 1997 Society for Neuroscience $\quad 0270-6474 / 97 / 174293-09 \$ 05.00 / 0$ terminals of different motoneurons at each individual junction. It is worth noting that at developing neuromuscular junctions, as in other systems, a neuron withdraws only a subset of its axonal terminals and maintains others. This raises an interesting question, whether the growth or retention of an individual terminal affects the retraction of sibling terminals. ("Sibling" will be used in this paper to refer to nerve terminals or branches of the same neuron.)

Several previous observations have provided evidence suggesting that different branches of the same neuron do not grow independently, but rather at the expense of one another (Devor and Schneider, 1975; Murphey and Lemere 1984; Smalheiser and Crain, 1984; Goldberg and Schacher, 1987; Gan and Macagno, 1995a). Therefore, a particular branch may be influenced to stop growing and to retract not only by inhibitory factors in its local environment, but also by strong competition from its more vigorously growing siblings. Indeed, in many developing systems, the retraction of some axonal branches has often been seen to be accompanied by growth of other branches (Innocenti, 1981; Stanfield and O'Leary, 1985; Gao and Macagno, 1987a,b; Jellies et al., 1987; Baptista and Macagno, 1988; Callaway and Katz, 1990; Lamantia and Rakic, 1990). Whether the outgrowth of some axonal branches does or does not contribute to the retraction of their sibling branches, however, has not been thoroughly tested experimentally thus far.

In this study, we specifically examined the role of competitive interactions among sibling branches in the retraction of specific neurites by an identified central neuron, the AP cell, in the medicinal leech (Hirudo medicinalis). The AP cell has longitudinal projections within anterior and posterior connective nerves and lateral projections that extend to the periphery 


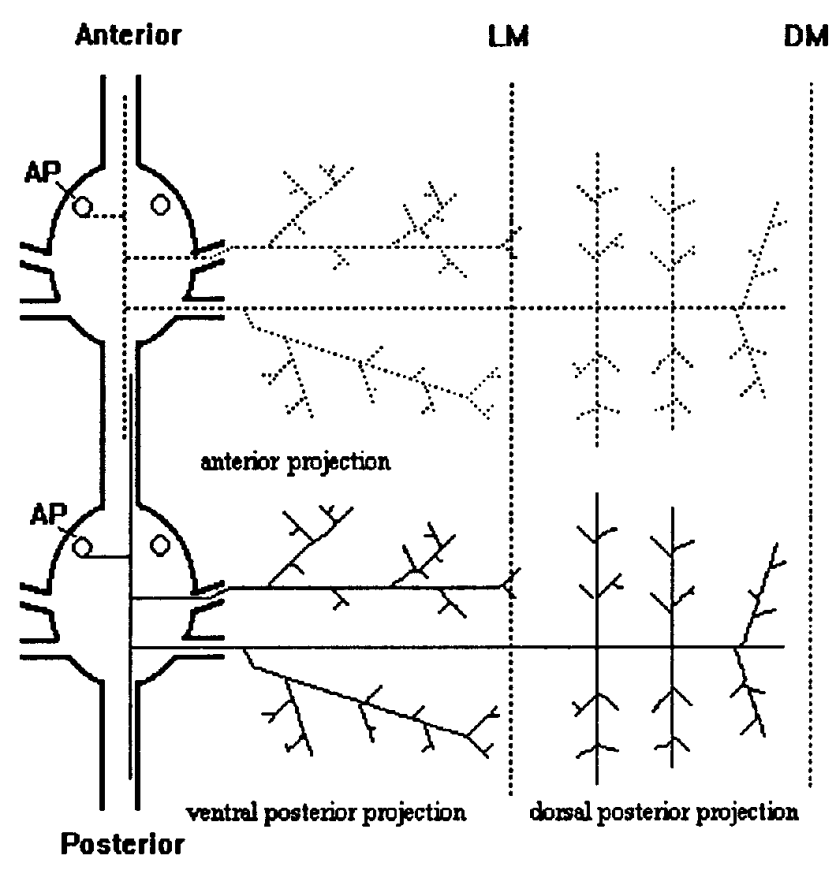

Figure 1. Schematic diagram of two AP cells in adjacent ganglia at E12-E13. Each AP cell has two lateral projections (Anterior and Posterior) that exit the ganglion via the contralateral nerve roots to the periphery, where they branch profusely. The posterior lateral projection further bifurcates into the ventral posterior projection and the dorsal posterior projection. In addition, each AP cell has two longitudinal projections that overlap with those of adjacent homologs within the connective nerves. $L M$, Lateral midline; $D M$, dorsal midline. Anterior is at the top in this figure, as well as in Figures 4 and 7.

through both ganglionic nerve roots (see Fig. 1). Previous studies have also determined that the lateral projections branch profusely in the periphery after embryonic day 10 (E10) (Gan and Macagno, 1995b), whereas the longitudinal projections eventually retract during late embryogenesis (Gao and Macagno, 1987b). Ablating the homologs of an AP neuron in adjacent ganglia, however, prevents this retraction and induces the longitudinal projections to grow into the periphery through adjacent ganglia (Gao and Macagno, 1987b). A very similar response by the longitudinal projections can be induced by cutting the two nerve roots that contain its lateral projections at a time when these lateral projections are innervating peripheral targets (Gao and Macagno, 1988).

In the experiments described in this paper, by varying the extent of outgrowth of either the lateral or the longitudinal projections using different paradigms, we provide several lines of experimental evidence in support of the idea that the lateral and longitudinal branches of the AP cell grow at the expense of each other, and that the retraction of the longitudinal branches is, in part, a consequence of the extensive outgrowth of the lateral branches in the periphery.

\section{MATERIALS AND METHODS}

Animals. H. medicinalis embryos were obtained from our laboratory colony and maintained at $23^{\circ} \mathrm{C}$. We staged embryos according to the criteria proposed by Fernandez and Stent (1982).

Cutting the lateral projections with a laser microbeam. Embryos were anesthetized with $9 \%$ ethanol in sterile artificial pond water $(0.5 \mathrm{gm} / 1$ Instant Ocean, Menasha Corporation) and were positioned in a groove cut into a SYLGARD-coated (Dow Corning, Arlington, TN) microslide. To identify the AP cells and fill them with dye, a small patch of skin over the experimental ganglion was removed using a sharp pin. In these experiments, we always examined AP neurons in the ganglia of midbody segments 10-16 (MG10-16). To cut specific processes of an AP neuron with the laser microbeam, the cell was first filled with the fluorescent dye 1,1'-dioctadecyl-3,3,3',3'-tetramethylindocarbocyanine perchlorate (DiI; Molecular Probes, Eugene, OR), made up to a concentration of $1 \%$ in methylene chloride (Sigma, St. Louis, MO). Somata were penetrated with dye-filled microelectrodes (resistance, $\sim 100 \mathrm{M} \Omega$ ) under a Zeiss (Thornwood, NY) $40 \times$ water immersion objective, and a depolarizing current (1 $\mathrm{nA}, 1 \mathrm{~Hz}$ ) was applied for a few seconds until a tiny crystal formed at the tip of the electrode. The electrode was then removed and the DiI crystal remained on or inside the cell body. The embryos were then placed into fresh sterile artificial pond water to recuperate (the wound seals rapidly) and to allow the DiI to diffuse throughout the arbor of the cell. A few hours later, a small patch of skin was again cut over the experimental ganglion, and the projections within the lateral roots were illuminated with a focused laser microbeam for 1-2 min, until the processes acquired a beaded appearance.

The $528 \mathrm{~nm}$ line of a $15 \mathrm{M} \Omega$ argon laser was used to ablate branches of DiI-filled cells in some experiments. The laser beam was focused through the $40 \times$ water immersion objective, generating a $1-2 \mu \mathrm{m}$ spot at the focal plane. The actual size of the spot at the specimen was somewhat larger because of the refractile properties of the living tissues through which the light traveled.

Cutting the roots. The anesthetized embryos were first positioned as described above. A sharp pin was used first to penetrate the skin above the roots under the dissecting microscope. The roots were then cut with the pin. After surgery, the embryos were returned to sterile pond water.

There was no regeneration of posterior lateral projections when the cut was made at E9-E10. When cutting at E11-E12, however, there were 4 of 21 cases in which the regeneration of posterior lateral projections occurred. The regenerated ones were thinner and occupied much smaller territories than their contralateral homologs. We did not distinguish the cases in which regeneration did occur versus no regeneration when we quantified the effect of cutting the posterior roots on the growth of longitudinal projections.

Cell ablations. Cell ablations were performed as in Gan and Macagno (1995a). Briefly, the identified AP cells were penetrated by microelectrodes $(100 \mathrm{M} \Omega$ ) filled with $1 \% 5(6)$-carboxyfluorescein (Sigma) in $0.2 \mathrm{M}$ $\mathrm{KCl}$. The dye was iontophoresed using negative pulses $(1 \mathrm{nA}$ at $1 \mathrm{~Hz}$ for $3 \mathrm{~min}$ ). The injected AP cells and parts of their branches were illuminated with a Xenon arc lamp for $1 \mathrm{~min}$. Cell death, as indicated by a swollen soma and beaded structures along the axons, ensued within a few hours.

DiI staining and imaging of the AP neuron. The procedures for staining and imaging have been described in detail in a previous publication (Gan and Macagno, 1995a). Briefly, anesthetized embryos were first opened dorsally and freed of yolk, then cut along the ventral midline to expose the ganglia of interest. A small DiI crystal was then injected into the AP cell body as described above.

After staining, preparations were immediately fixed in $4 \%$ paraformaldehyde, maintained in this solution at room temperature for 2-7 d, and mounted on a slide for observation and study. Images were taken with a confocal microscope (MRC-600, Bio-Rad, Richmond, CA) by optically sectioning the embryo and then superimposing the optical sections to generate the final images.

Quantification of the outgrowth of the AP longitudinal and lateral projections. The length of the AP longitudinal projections relative to the interganglionic length of the connective nerve was used to quantify its outgrowth (Fig. $2 A$, diagram). Such a method of measurement avoids possible variations inherent in the manual stretching of the preparation during dissection and fixation. The length of the longitudinal projection was measured from the edge of the ganglion to the tip of the growth cone, and the length of the connective nerve was measured from the anterior edge of one ganglion to the posterior edge of its neighbor in the anterior neighboring segment.

The AP cells follow axonal branches of the $\mathrm{P}_{\mathrm{D}}$ cells in the dorsal germinal plate and form six first-order branches approximately perpendicular to the main projection, similar to the branching pattern of the $P_{D}$ cell (Fig. 1). By taking advantage of the regular growth pattern of AP lateral projections, we quantitated the amount of growth of the AP cell in the dorsal germinal plate in the same way as we quantified previously the growth of $P_{D}$ cells (Gan and Macagno, 1995b). The growth of each of the six first-order branches was measured in terms of the number of annuli it covered from its initiation on the main projection to the furthest anterior or posterior extension of its high-order branches. (There are five annuli per segment in the midbody of $H$. medicinalis.) The amount of growth of 
A

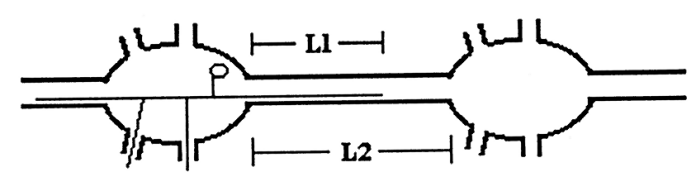

Relative length $=\mathrm{L} 1 / \mathrm{L} 2$
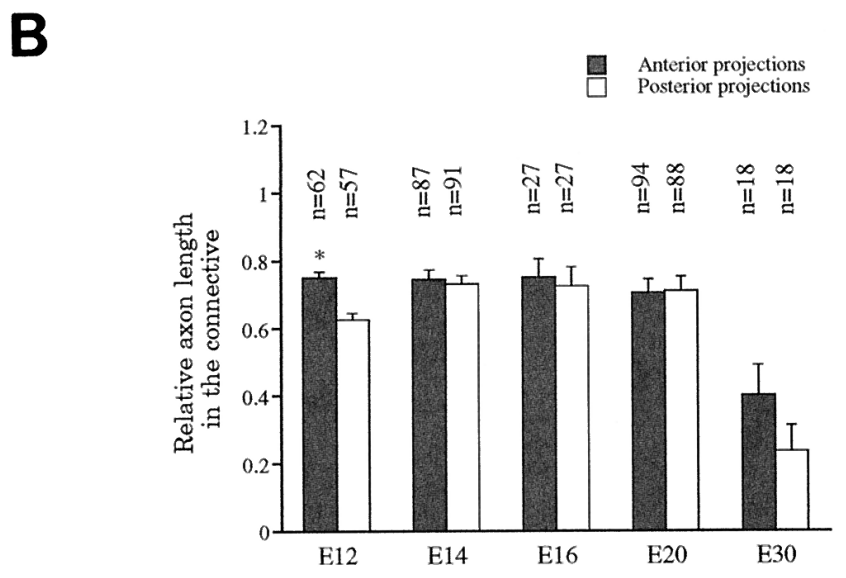

C

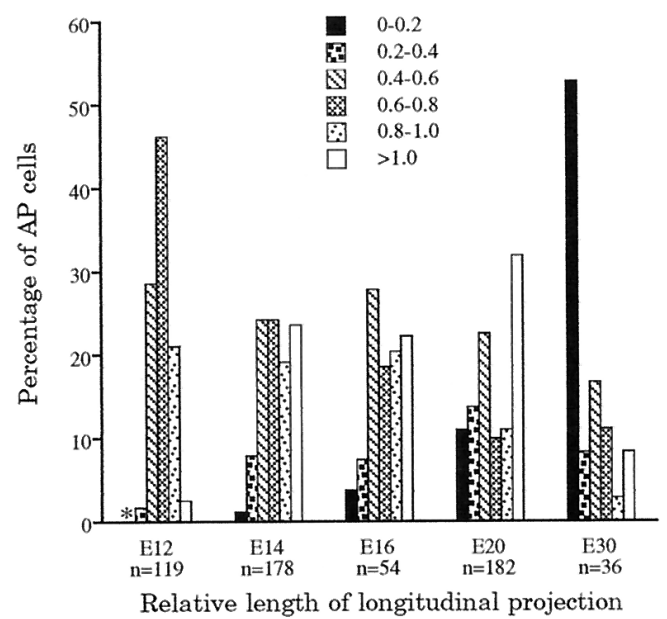

Figure 2. Relative lengths of the longitudinal projections as a function of time in development. $A$, Diagram showing the parameters measured to obtain the relative lengths of the AP longitudinal projections in the connective nerve between adjacent ganglia (L1/L2). B, Comparison of relative lengths in the posterior and anterior directions at different stages. Only at E12 are the differences in relative length significant, with the anterior projections $\left({ }^{*}\right)$ slightly longer $(p<0.005)$. Between E12 and E20, average relative lengths remain the same, but a large reduction was found at E30. In the adult, these projections are effectively absent. $C$, The distribution of relative lengths changes with embryonic age. Anterior and posterior longitudinal projections were not distinguished here. The asterisk associated with the left-most data point at E12 denotes that there are no longitudinal projections with relative lengths between 0 and 0.2 at this stage.

the AP lateral projection in the dorsal germinal plate was then calculated as the sum of the extension of all six first-order branches. Such a method of measurement has the advantage that it also normalizes for possible variations in the stretching of the preparation during dissection and fixation.

\section{RESULTS}

\section{The extension and retraction of AP longitudinal projections is a protracted process that occurs over several weeks}

The average relative length of the anterior and the posterior longitudinal projections remained approximately the same from E12 to E20, but decreased by more than half from E20 to E30 (0.7 to $\sim 0.3)$ (Fig. $2 B)$. Because the absolute length of the connective nerve increased $\sim 1.5$-fold from $\mathrm{E} 12(n=58)$ to E20 $(n=84)$ and $\sim 1.6$-fold from E20 $(n=84)$ to E30 $(n=18)$, the average absolute length of the longitudinal projections actually increased from E12 to E20 but then decreased sometime between E20 and E30.

At any one embryonic stage, the length of the longitudinal projections varied greatly among AP cells in different animals or in different segments within an animal. For the 119 AP cells examined at E12, for example, the relative lengths of these projections showed a bell-shaped distribution (Fig. 2C), with the largest fraction having relative lengths of $0.6-0.8$. As a function of developmental age, this distribution changed significantly in shape. Interestingly, both the very short and very long projections increased in number between E12 and E20, indicating that some projections retracted, whereas others continued to grow throughout this period. By the end of embryogenesis (E30), 33\% of the longitudinal projections had retracted entirely and many others were quite short, but $\sim 8 \%$ still extended into adjacent ganglia. Because longitudinal projections are almost never found in adults, and never extending as far as adjacent ganglia, process retraction must continue beyond the end of embryogenesis.

Our observations show that the extension and retraction of longitudinal projections by AP neurons occurs in a time frame of several weeks. In addition, when a particular process will stop growing and begin to retract seems unpredictable, although the distributions of relative lengths versus embryonic age suggest that longer projections, particularly those that reach the adjacent ganglia, may be relatively more stable. The probability of retraction can be strongly modulated, however, by the extent of growth of the lateral projections of the AP cell in the periphery, as shown by the results of the experiments that follow. The posterior longitudinal projection grows faster than
the anterior one after the cutting of both lateral roots

As reported in a previous study (Gao and Macagno, 1988), cutting both of the nerve roots that contain the lateral projections of an AP neuron induces its longitudinal projections to grow into the periphery through adjacent ganglia. To get a better understanding of the dynamics of this response, we cut both lateral roots at E12-E13 and subsequently examined the longitudinal projections of the experimental AP cells at several time points. The intact contralateral AP homologs in the same ganglia served as controls.

Neither the anterior nor the posterior longitudinal projections showed significant growth relative to controls $7-8 \mathrm{hr}$ after the operation ( $p>0.4 ; n=20$; Fig. 3). After another 7 hr, however, both projections were significantly longer in the experimental AP cells. For the anterior projection, the relative length increased $26 \%$ over controls, from $0.77 \pm 0.07$ (mean \pm SEM) to $0.97 \pm$ $0.06(n=14 ; p<0.02)$, whereas for the posterior longitudinal projections, this value increased $51 \%$, from $0.73 \pm 0.06$ (control) to $1.1 \pm 0.05(n=16 ; p<0.001)$ (Fig. 3). The change in the posterior projection was significantly greater than that in the anterior one $(p<0.05 ; n=20)$. Seven hours later this effect was even more pronounced; by this time the increase in relative length 


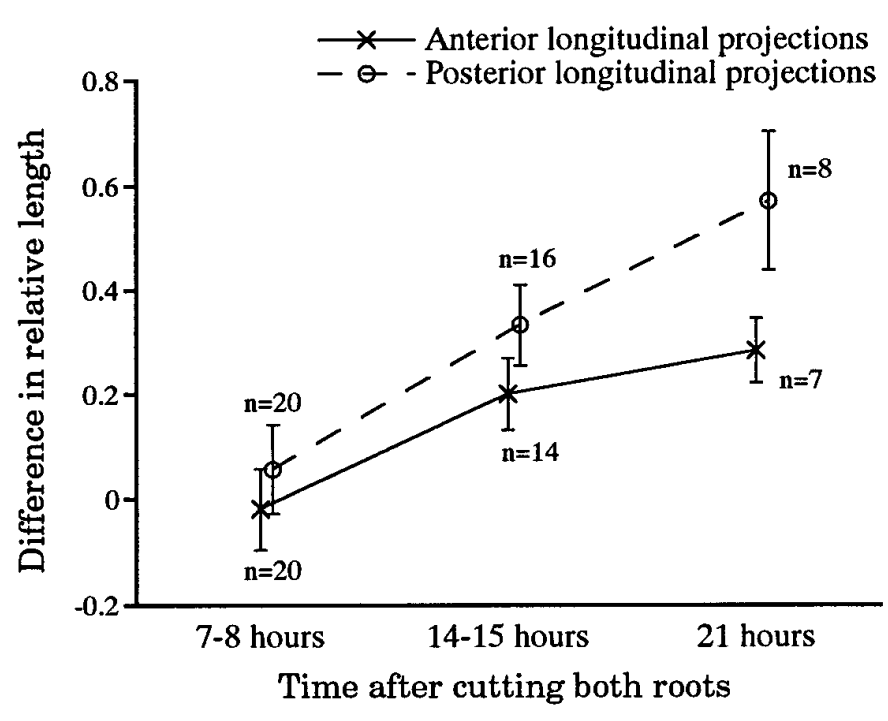

Figure 3. Cutting both nerve roots greatly enhances the growth of the longitudinal projections of the AP cell. The difference in relative length was calculated as the difference in relative length (as defined in Fig. $2 A$ ) between the longitudinal projections of the experimental AP neuron (the lateral projections of which were cut) and those of the intact contralateral homolog in the same ganglion. By 14-15 hr after root cutting, the relative lengths of both the anterior and posterior longitudinal projections of the experimental cells were significantly longer than controls. The posterior longitudinal projection grew significantly more than the anterior one at both $14-15 \mathrm{hr}$ and $21 \mathrm{hr}$ after the surgery.

of the anterior projection was $\sim 39 \%$, whereas the posterior projection showed an increase of $\sim 78 \%$ (Fig. 3). By then both longitudinal projections had reached or were about to exit from the adjacent ganglia. As we discuss later, the greater early response of the posterior longitudinal projection may well reflect local effects of reducing lateral outgrowth.

\section{Cutting both lateral projections with the laser microbeam causes the longitudinal projections to grow into the periphery through adjacent ganglia}

Because many axons beside those of the AP cell are severed when the ganglionic nerve roots are cut as described above, it is possible that the response of enhancing longitudinal extension of the AP cell is triggered by some factor other than the loss of its lateral projections. To test whether cutting just the lateral projections of the AP cell has the same effect, we used a laser microbeam to cut the lateral projections of a series of AP neurons several hours after they had been injected with DiI (see Materials and Methods). These experiments were performed at E12-E13, when the lateral projections are growing vigorously, but many of the longitudinal ones have already slowed down or stopped their extension along the connective nerves. Two days after the laser surgery, in all of the 12 cases examined, the longitudinal projections were found to have grown into the periphery via the adjacent ganglia; one of the experimental AP cells is shown in Figure 4.

Two controls were performed for these experiments. First, to test for possible effects of dye filling, six AP cells were stained with DiI but not irradiated; they were examined 2 d later. Second, to test for effects of possible damage to other components of the nerves, both lateral nerves containing no stained AP projections were illuminated with the focused laser microbeam for $6 \mathrm{~min}$ (three times as long as required to ablate stained AP projections) in six different ganglia of three animals. The AP cells in these ganglia were stained with DiI 2 d later. None of the AP cells subjected to these two protocols was found to have extended longitudinal projections into the periphery of adjacent segments. In addition, we also performed the experiment in which five AP cells were stained with DiI, but only one of their two lateral projections was ablated (three posterior and two anterior ones). In none of these five cases were the longitudinal projections found to extend beyond the adjacent ganglia into the periphery.

We conclude that extension of longitudinal projections into the periphery is a specific effect of laser cutting both lateral projections of a developing AP neuron.

\section{Partially reducing the outgrowth of the lateral projections also induces additional growth of the longitudinal axons}

Cutting only the posterior root can also induce greater outgrowth of the longitudinal projections. We first cut the posterior nerve root in ganglia of E9-E10 animals, at a time when the posterior lateral projection of the AP neuron has just entered the periphery but has not yet branched vigorously. In 14 cases examined $4 \mathrm{~d}$ after cutting, the relative length of the posterior longitudinal projections of the experimental AP neurons was found to be $1.02 \pm 0.04$ (mean \pm SEM), significantly greater $(p<0.005 ; n=14)$ than that of the control contralateral homologs $(0.77 \pm 0.05)$. The anterior longitudinal projections, however, did not show a statistically significant effect $(p>0.05 ; n=14)$ in this experiment (Fig. 5).

A somewhat different result was obtained when the posterior root was cut at E11-E12, a time when the posterior lateral projections were already elaborating extensive arbors in the periphery. Four days after this operation, both anterior and posterior longitudinal projections showed significantly greater outgrowth than the controls $(p<0.005 ; n=21)$ (Fig. 5). The posterior root seldom regenerated (4 cases of 21), and the territory vacated was usually invaded by the intact anterior lateral projection of the AP cell. In 2 of 21 cases, a displaced thin posterior lateral projection was found to exit along the anterior root into the periphery (data not shown).

It is worth noting that after cutting the posterior root, the longitudinal projections never grew into the periphery through adjacent ganglia although they grew longer than the controls. We did not perform a series of anterior root cuts, because previously reported observations suggested that cutting either root would yield the same results (Gao and Macagno, 1988).

\section{Ablation of the dorsal P cell, which reduces severely the growth of the posterior lateral projection, enhances the growth of the posterior longitudinal projection}

After exiting through the posterior root, the posterior lateral projection of the AP cell bifurcates into two branches that extend along the dorsoposterior (DP) and ventroposterior (VP) nerves. These branches then branch again extensively to innervate the corresponding areas in the body wall. In a previous study, we reported that the peripheral projections of the AP cell grow very accurately along the earlier-growing peripheral arbors of the contralateral ventral and dorsal $\mathrm{P}$ cells (Gan and Macagno, 1995b). In addition, we found that ablating the dorsal $\mathrm{P}$ cell reduced significantly the arborization of the DP projection of the AP cell, whereas the VP projection remained essentially intact. Here, we were specifically interested in whether reducing the outgrowth of the DP lateral projection of the AP cell by ablating the dorsal $\mathrm{P}$ cell might also induce additional growth of its longitudinal projections. 


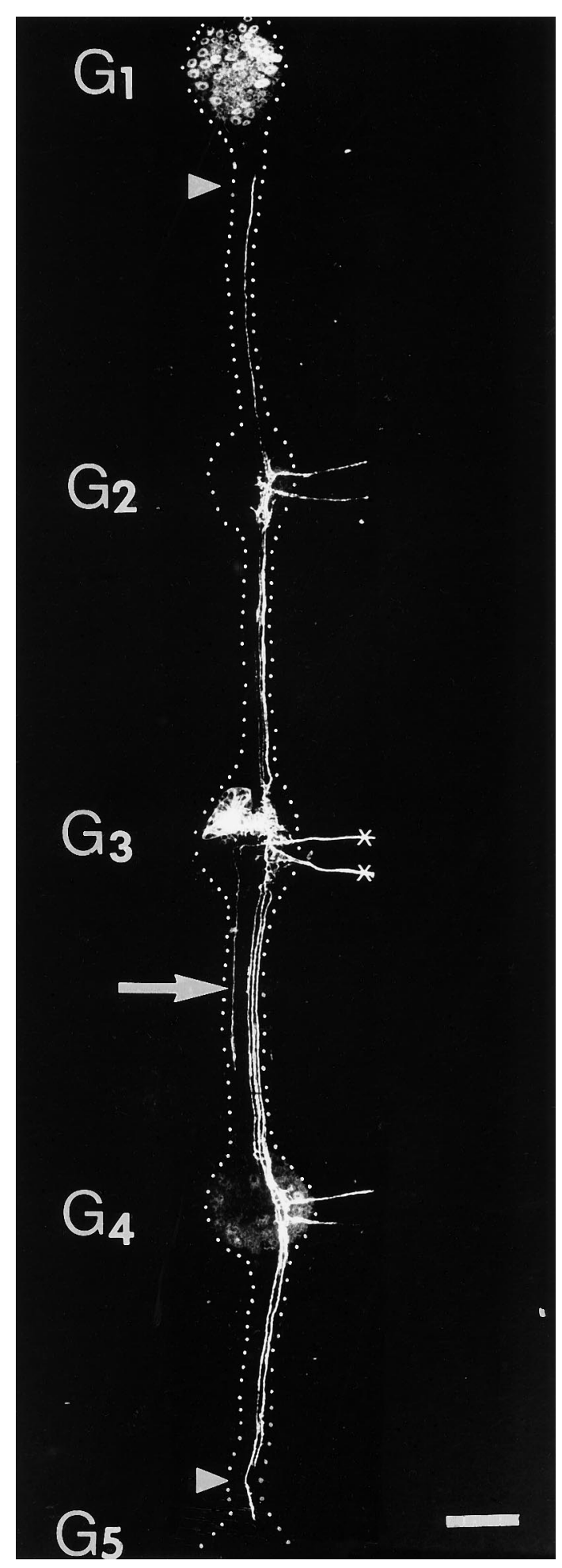

Figure 4. Example of the effect of cutting both lateral projections (crosses) with a laser microbeam at E12. Two days after the operation, both anterior and posterior longitudinal projections had grown into the periphery of adjacent segments in this preparation. In addition, the longitudinal projections had grown past the adjacent ganglia $(G 2$ and $G 4)$ and had almost reached the next ones (G1 and G5) (arrowheads). This cell had collateral projections, which can be seen in the interganglionic nerves, a feature that can also be found in some normal cells. This cell, however, also extended a novel ipsilateral longitudinal projection (arrow) in the posterior direction; ipsilateral projections were never seen in controls. Dotted lines added to outline the boundaries of the ganglia and interganglionic connectives. Scale bar, $200 \mu \mathrm{m}$.

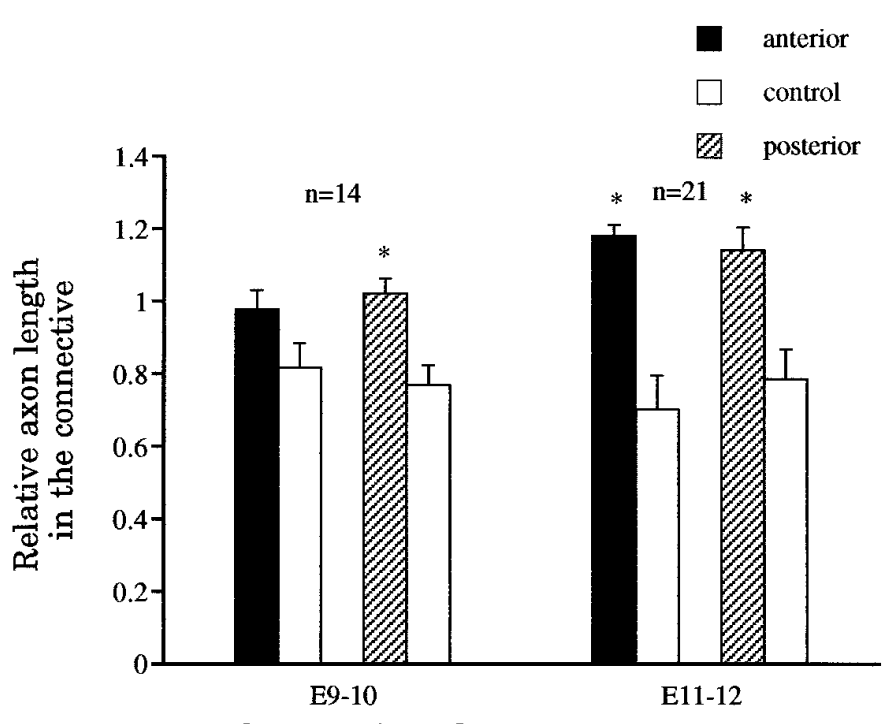

Embryonic day when cutting posterior roots

Figure 5. Cutting only the posterior nerve root also enhances the growth of the longitudinal projections. Four days after cutting the posterior root at either E9-E10 or E11-E12, the longitudinal projections of the experimental cells $(*)$, with the exception of the anterior longitudinal projection when cutting was performed at E9-E10, had grown significantly more than controls. In contrast, however, to the effects of cutting both nerve roots or cutting both lateral projections with the laser microbeam (see Fig. 4), here the longitudinal projections did not grow beyond adjacent ganglia or out to the periphery from them.

Ablating the dorsal P cell between E8 and E9 did not induce significantly more outgrowth ( $p>0.2 ; n=18$ ) of either anterior or posterior longitudinal projections of the AP cell by E12, a time when the AP cell has just begun vigorous arborization in the periphery (Fig. 6). By E14, however, the posterior longitudinal projection of the AP cell was found to have significantly greater outgrowth than the control $(p<0.01 ; n=29)$, whereas the anterior longitudinal projection showed no significant effects $(p>$ $0.2 ; n=29$ ). Not unexpectedly, the increased outgrowth of the posterior longitudinal projection induced by reducing the dorsal arbor of the posterior lateral projection is clearly smaller than the effect produced by cutting the posterior root, which eliminated both the dorsal and the ventral arbors of the posterior lateral projection.

\section{Intact lateral projections have smaller arbors when the longitudinal projections are induced to grow into the periphery of adjacent segments by the ablation of segmental homologs of the AP neuron}

The results described above show that reduced outgrowth of the lateral projections under various conditions enhances the outgrowth of the longitudinal projections. As a complementary experiment, we asked whether inducing greater outgrowth of the longitudinal projections reduces the outgrowth of intact lateral projections.

Previous studies have shown that the longitudinal projections of the AP cell will grow into the periphery of adjacent segment within $2 \mathrm{~d}$ after ablating the adjacent AP homologs at E9-E12 (Gao and Macagno, 1987b). We repeated these experiments here but concentrated our analysis on the peripheral growth of the lateral projections. Two days after killing, at E9-E10, the AP homologs in two adjacent anterior and two adjacent posterior ganglia, we observed that the lateral projections of the experimen- 


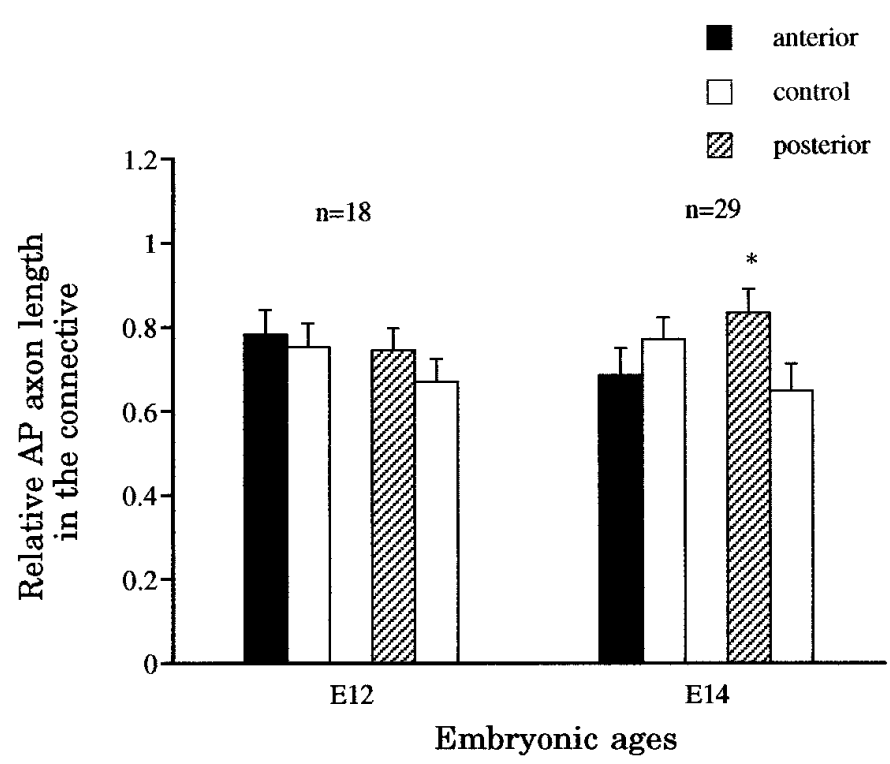

Figure 6. Effect of ablating a $\mathrm{P}_{\mathrm{D}}$ cell on the outgrowth of the AP longitudinal projections. The ablation was performed at E8-E9, but the embryos were examined at E12, when the lateral projections of the AP cell are initially established, or E14, when normally there is already an extensive arbor. At E12, neither the anterior nor the posterior longitudinal projections were significantly longer than the controls $(p>0.2)$. At E14, however, the posterior longitudinal projections $(*)$, but not the anterior ones, had grown significantly more than the controls $(p<0.01)$.

tal AP neuron had reduced arbors in the periphery relative to controls, whereas the longitudinal branches now extended into the periphery through the adjacent ganglia (Fig. $7 A$ ). The reduction of the AP terminal arborization can be observed in the ventral as well as the dorsal germinal plate, but it is much more obvious in the dorsal region. An example of this is shown in Figure 7, $B$ and $C$, which show, respectively, the control and experimental arbors of AP cells in the dorsal germinal plate $4 d$ after homolog deletion. The longitudinal projections of the experimental AP cell grew extensively in the dorsal region of the adjacent segments (Fig. 7C), whereas its lateral projection was reduced greatly in the dorsal germinal plate compared with that of its contralateral homolog (Fig. 7B).

To get a quantitative assessment of this effect, we made the following measurement. Because the DP projection of the AP cell in the dorsal germinal plate forms three first-order branches that are approximately perpendicular to the shaft of the DP projection, we estimated the total length of the six first-order branches in units of annular width as a measure of the amount of outgrowth of the DP projection (Fig. 1 schematic and Materials and Methods). Figure $7 D$ shows that $2 \mathrm{~d}$ after either one or both longitudinal projections had grown into adjacent segments because of the ablation of adjacent homologs, the total length of the six firstorder branches of the DP projection was significantly shorter in experimental cells than in the controls $(p<0.005)$.

In all the cases examined, visual inspection of the dye-stained arbors gave the impression of an inverse relation between the amount of outgrowth of the longitudinal projections in adjacent segments and the extent of reduction of the lateral arbor. Because of the great difficulty in measuring the size of terminal arbors, however, especially in the ventral region, we did not confirm this apparent inverse relationship quantitatively.

\section{DISCUSSION}

The observations reported here extend significantly previous findings on the outgrowth of the AP cell (Gao and Macagno, 1987b; 1988). We demonstrate here that the lateral and longitudinal projections of the AP cell do not grow independently of one another but, rather, at the expense of each other. Furthermore, our results show that such competition among the projections of the AP cell plays a critical role in preventing the longitudinal projections from growing through adjacent ganglia to form permanent arbors in the periphery. We propose, therefore, that sibling neurite competition is normally a key component, along with inhibition by the AP homolog (Gao and Macagno, 1987b), of the process that culminates in the retraction of the AP longitudinal projections. Mechanisms such as this one may play similar roles in defining the patterns of neural arborization in other developing nervous systems.

\section{Sibling projections compete with one another, limiting the growth of each other}

We used four different experimental manipulations to demonstrate that reducing or eliminating the growth of the lateral projections enhances the growth of the longitudinal projections: (1) cutting both nerve roots; (2) cutting only the lateral projections of the AP cell in the two nerve roots, with a laser microbeam; (3) cutting only the posterior nerve root; and (4) ablating the dorsal P cell (Fig. 8). One interesting observation from all these manipulations is that the more the outgrowth of the lateral projections is reduced, the more the outgrowth of the longitudinal projections increases. In the first three cases in which the lateral projections were cut (manipulations 1-3), the observed effect was greater outgrowth of one or both of the longitudinal projections. This approach, however, leaves open the possibility that the enhanced growth of the longitudinal projections is a consequence of cell damage. Axotomy-induced responses, such as changes in protein synthesis or increases in excitability (Giulian et al., 1980; Goldberg and Ambron, 1986; Simoni et al., 1990), might also influence the growth of the longitudinal projections. This concern, however, is allayed by the results of experiment 4. In this case, there was no damage to the AP neuron itself, because the reduced growth of the lateral dorsal arbor was an indirect response to the ablation of the pioneer $\mathrm{P}_{\mathrm{D}}$ cell used by the AP cell as a template to construct its dorsal arbor (Gan and Macagno, 1995b). Considered together, the results of all four kinds of experiments suggest strongly that the lateral projections compete with the longitudinal projections for something required for neurite outgrowth.

A final line of experimental evidence supporting this conclusion is provided by the converse experimental protocol, directly enhancing the growth of the longitudinal projections instead of perturbing the growth of the lateral projections. Increasing the growth of the longitudinal branches by ablating the adjacent homologs leads to a significant reduction in the growth of the lateral branches. There was no damage to the AP cell in this experiment, the response to the perturbation being indirect. All the results we have obtained, therefore, are consistent with one another and lead to the same conclusion.

The growth of the lateral projections seemed to influence the outgrowth of the anterior and posterior longitudinal projections differentially. When both nerve roots were cut, for example, posterior longitudinal projections showed more extensive growth than did anterior ones 14-15 hr after the surgery. Furthermore, the posterior longitudinal projections, but not the anterior ones, showed significant growth relative to controls $4 \mathrm{~d}$ after the cutting 

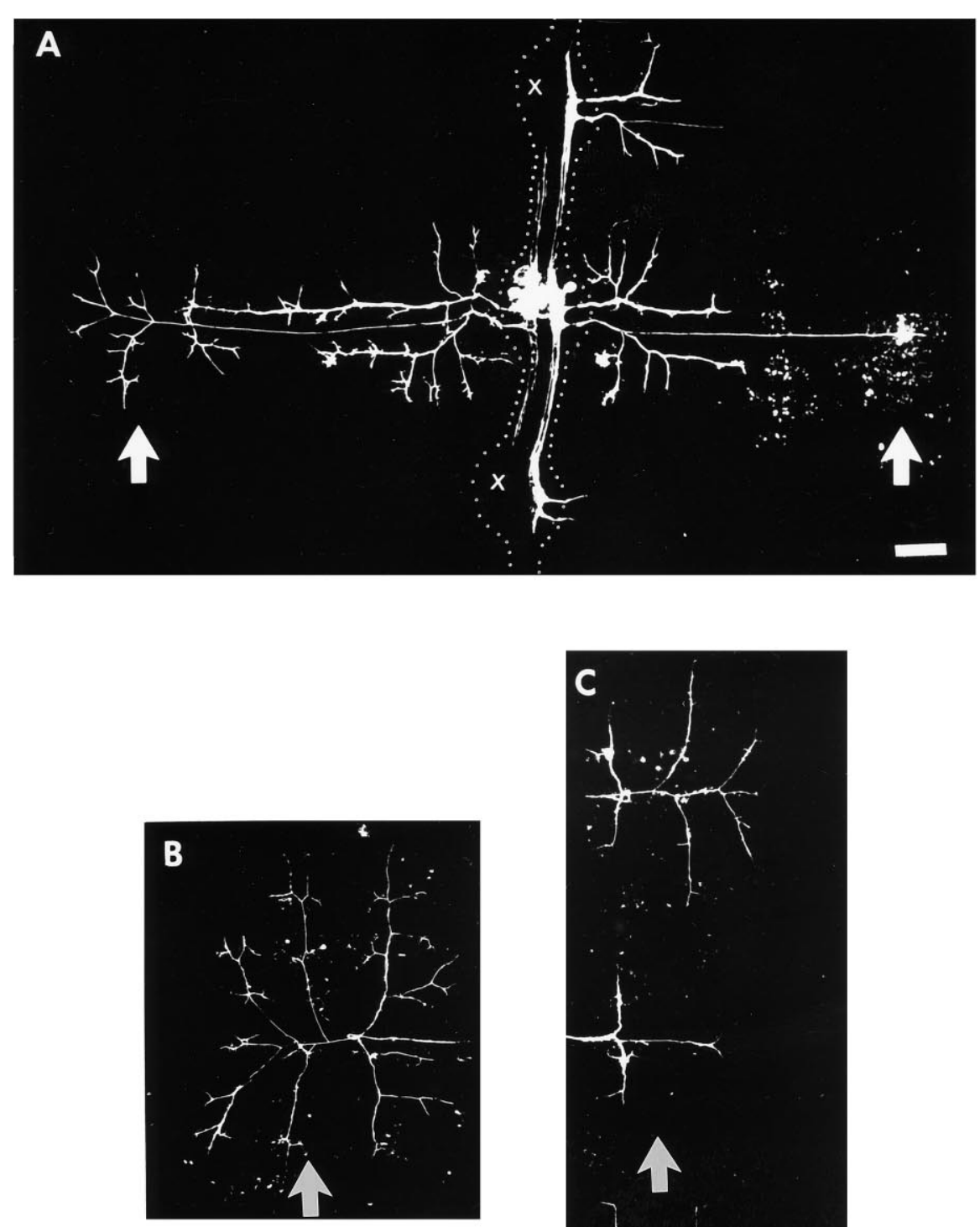

D

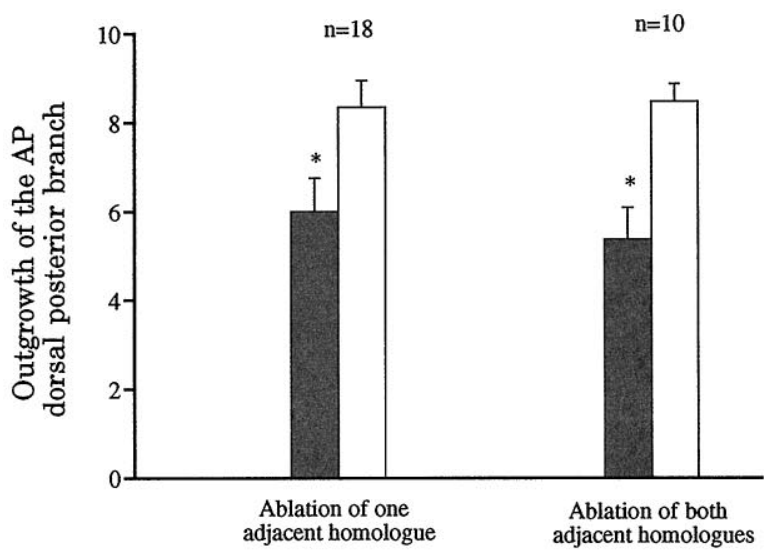

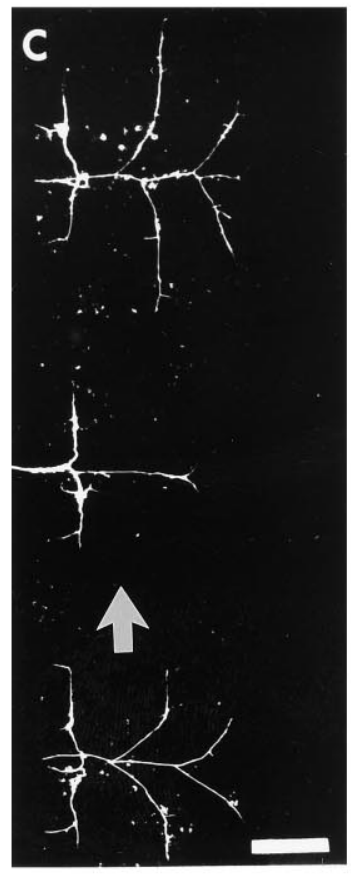

Experimental

Control djacent homologues
Figure 7. Greater outgrowth of its longitudinal projections reduces the arborization of the lateral projections of the AP cell. $A$, In this preparation, four AP cells were ablated at E9-E10 on the same side in the two ganglia adjacent to the ganglion of interest (crosses) as well as the next two ganglia (not shown). Two days later, the experimental AP cell on the same side as the ablated AP cells had extended its longitudinal projections ectopically to the periphery, through the adjacent ganglia (on the right of the picture). At the same time, the lateral projections of this AP cell grew less in its own segment than those of its contralateral homolog, which serves as the control. The effect is more obvious in the dorsal germinal plate (arrows). Dotted lines were added to show boundaries of ganglia and interganglionic connectives. $B, C$, Another example, $4 \mathrm{~d}$ after ablation of the four adjacent ipsilateral AP cells, of the difference in outgrowth of control $(B)$ and experimental $(C)$ AP cells in the dorsal germinal plate (corresponding to the region in $A$ indicated by arrows). The longitudinal projections of the experimental AP cell had grown extensively in the dorsal region of the adjacent segments ( panel $C$ ), but the lateral projection had significantly reduced outgrowth in the dorsum of its own segment (arrow), compared with the contralateral homolog shown in $B$ (arrow). $D$, Quantitative measurements of the arbors of control and experimental AP cells in the dorsal germinal plate of their own segments. The data, taken $2 \mathrm{~d}$ after ablating either one AP cell located ipsilaterally in either an anterior or a posterior adjacent ganglion (left) or both adjacent ipsilateral AP cells (right), show 35 and $50 \%$ reductions in outgrowth, respectively (asterisks). Anterior is at the top in panels $A-C$. Scale bars, $100 \mu \mathrm{m}$; scale bar in $C$ also applies to $B$. 
A
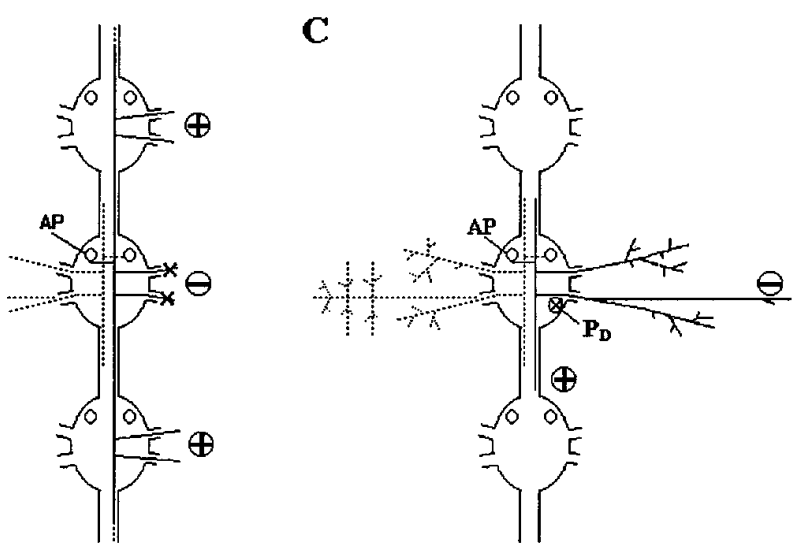

B

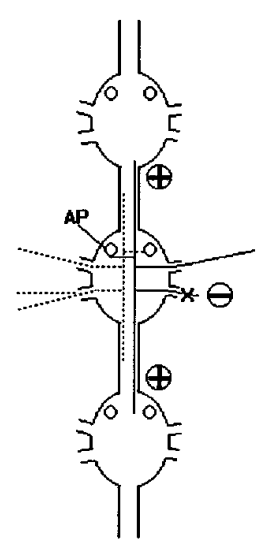

$\mathbf{D}$

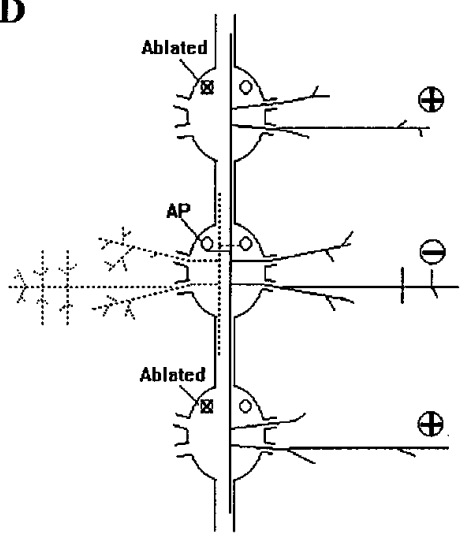

Figure 8. Schematic diagrams summarizing the results of the different types of protocols used in the experiments reported here. In each case, the circled sign indicates a reduction $(-)$ or an increase $(+)$ in the arbor of the experimental AP neuron. $A$, Cutting both roots or ablating both lateral projections $(-)$ leads to increased growth of longitudinal projections $(+)$. $B$, Cutting the posterior nerve root (-) causes additional growth of both longitudinal projections $(+)$, but neither of these grows sufficiently to exit to the periphery through the next ganglia. $C$, Ablating the $\mathrm{P}_{\mathrm{D}}$ cell $(X)$ that serves as a template reduces the dorsal arborization of the AP neuron $(-)$ and increases the growth of its posterior longitudinal projection (+). $D$, Ablating ipsilateral homologs $(X)$ results in enhanced growth of the longitudinal projections of an AP cell (+), but a diminution of its lateral arborization (-).

of the posterior root at E9-E10 in experiment 3 or $6 \mathrm{~d}$ after the ablation of the $\mathrm{P}_{\mathrm{D}}$ cell in experiment 4 . Because posterior longitudinal projections are physically nearer to the lateral projections than are anterior longitudinal projections (Fig. 1), these results suggest that closely situated branches may interact more strongly than branches located farther apart.

Competition among different branches of the same neuron has been proposed in several other systems (Schneider, 1973; Devor and Schneider, 1975; Murphey and Lemere, 1984; Gan and Macagno, 1995a). For instance, when retinal axons in newborn hamsters projected to the ipsilateral superior colliculus (SC) as well as to the thalamic nucleus lateralis posterior (LP) after lesions of the contralateral SC, the greater the amount of terminal arborization found in the SC, the less was found in the LP (Schneider, 1973). Recently, we have demonstrated at the single cell level that different branches of the dorsal $\mathrm{P}$ cell grow at the expense of each other in the periphery (Gan and Macagno, 1995a). Such interactions among sibling branches may reflect competition for a limited supply of materials, such as cytoskeletal elements or other com- ponents important for outgrowth, which are generated at the soma at rates that cannot support extensive growth of all branches (Schneider, 1973; Devor and Schneider, 1975; Smalheiser and Crain, 1984).

\section{Competition among sibling branches contributes to process retraction}

The results presented here, along with those of previous work (Gao and Macagno, 1987b; Wolszon et al., 1994a,b), demonstrate that two factors are necessary for the eventual retraction of the longitudinal projections of the AP neurons: (1) competitive interactions among sibling branches and (2) inhibitory interactions among homologs. Neither alone is sufficient to ensure retraction, because in the absence of either factor the longitudinal projections grow through adjacent ganglia and form permanent peripheral arbors.

How does the retraction of the AP longitudinal projections occur? Our results indicate that the retraction of the longitudinal projections happens within a time window of several weeks (Fig. 2). Interestingly, the longitudinal projections of many AP cells continue to grow in absolute length after E12, when the joint action of sibling neurite competition and inhibition by adjacent homologs keeps these projections from growing out to the periphery. Furthermore, even at the end of embryogenesis (E30), 8\% of the AP longitudinal projections were still extended into the adjacent ganglia. It seems unlikely, therefore, that homolog inhibition and sibling neurite competition directly induce the retraction of the longitudinal projections. It is possible that the role of inhibition and competition is to prevent the longitudinal projections from growing into the periphery and other factors eventually come into play to cause the actual retraction process. Various extrinsic growth-inhibiting factors, such as a $33 \mathrm{kDa}$ glycoprotein in retinal tectum (Stahl et al., 1990), collapsin (Luo et al., 1993), myelin-associated protein NI-35 (Schwab et al., 1993), netrin-1 (Colamarino and Tessier-Lavigne, 1995), and several neurotransmitters (Haydon et al., 1984; Mattson et al., 1988; McCobb et al., 1988) have been shown either to inhibit axonal outgrowth or to induce axon retraction in vitro. Recently, it was found that acetylcholine increased the probability of retraction of one specific branch of Retzius neurons in a developing glossiphoniid leech (Elsas et al., 1995). Similar factors might be involved in triggering the retraction of AP longitudinal projections within the connective nerve of the embryonic leech.

Process loss has been observed in many nervous systems or parts thereof, such as the corpus callosum (Innocenti, 1981; LaMantia and Rakic, 1990), corticospinal projections (Stanfield and O'Leary, 1985), horizontal connectional axons of the visual cortex (Callaway and Katz, 1990), and retinotectal axons (O'Rourke et al., 1994). Several leech neurons, other than the AP cell, trim some of their processes as well (Wallace, 1984; Glover and Mason, 1986; Gao and Macagno, 1987a,b; Jellies et al., 1987; Baptista and Macagno, 1988; Elsas et al., 1995). The selective retraction of some branches generally seems to occur at the same time as sibling branches are maintained or are growing extensively. For example, in the medicinal leech, the RPE neurons and the Retzius neurons of the fifth and sixth body segments retract axonal branches within the connective nerves as well as within the body wall when certain peripheral projections innervate reproductive tissues and begin to arborize extensively. Early ablation of the reproductive tissue not only eliminates the extensive arborization of those branches that normally innervate the target but also prevents the loss of sibling axons (Jellies et al., 1987; Loer et al., 
1987, 1989; Baptista and Macagno, 1988). Similarly, horizontal axon collaterals of pyramidal cells in the cat striate cortex do not connect precisely to the appropriate target early during development; instead, selective process elimination occurs later when axon collaterals branch extensively within the target area (Callaway and Katz, 1990). Another example is competition at the neuromuscular junction, where some axonal terminals are eliminated and their siblings are maintained (Redfern, 1970; BaliceGordon and Lichtman, 1993). As is the case for the AP neurons, competition among sibling neurites may contribute to process retraction in each of these different systems.

\section{REFERENCES}

Balice-Gordon RJ, Lichtman JW (1993) In vivo observations of pre- and postsynaptic changes during the transition from multiple to single innervation at developing neuromuscular junctions. J Neurosci 13:834-855.

Baptista CA, Macagno ER (1988) Modulation of the pattern of axonal projections of a leech motor neuron by ablation or transplantation of its target. Neuron 1:949-962.

Callaway EM, Katz LC (1990) Emergence and refinement of clustered horizontal connections in cat striate cortex. J Neurosci 10:1134-1153.

Colamarino SA, Tessier-Lavigne M (1995) The axonal chemoattractant netrin-1 is also a chemorepellent for trochlear motor axons. Cell 81:621-629.

Colman H, Lichtman JW (1993) Interactions between nerve and muscle: synapse elimination at the developing neuromuscular junction. Dev Biol 156:1-10.

Devor M, Schneider G (1975) Neuroanatomical plasticity: the principle of conservation of total axonal arborization. In: Aspects of neural plasticity, Vol 43 (Vital-Durand F, Jeannerod M, eds), pp 191-200. Paris: INSERM Colloquia.

Elsas SM, Kwak EM, Stent GS (1995) Acetylcholine-induced retraction of an identified axon in the developing leech embryo. J Neurosci 15:1419-1436.

Fernandez J, Stent GS (1982) Embryonic development of the hirudinid leech Hirudo medicinalis: structure, development and segmentation of the germinal plate. J Embryol Exp Morphol 72:71-96.

Gan WB, Macagno ER (1995a) Interactions between segmental homologues and between isoneuronal branches guide the formation of sensory terminal fields. J Neurosci 15:3243-3253.

Gan WB, Macagno ER (1995b) Developing neurons use a putative pioneer's peripheral arbor to establish their terminal fields. J Neurosci 15:3254-3262.

Gao WQ, Macagno ER (1987a) Extension and retraction of axonal projections by some developing neruons in the leech depends upon the existence of neighboring homologues. I. The HA cells. J. Neurobiol 18:43-59.

Gao WQ, Macagno ER (1987b) Extension and retraction of axonal projections by some developing neurons in the leech depends upon the existence of neighboring homologues. II. The AP and AE cells. J Neurobiol 18:295-313.

Gao WQ, Macagno ER (1988) Axon extension and retraction by leech neurons: severing early projections to peripheral targets prevents normal retraction of other projections. Neuron 1:269-277.

Giulian D, Des Ruisseaux H, Cowburn D (1980) Biosynthesis and intraaxonal transport of proteins during neuronal regeneration. J Biol Chem 255:6494-6501.

Glover JC, Mason A (1986) Morphogenesis of an identified leech neuron: segmental specification of axonal outgrowth. Dev Biol 115:256-260.

Goldberg DJ, Ambron RT (1986) Consequences of partial axotomy for production of neurotransmitter vesicles and routing of rapidly transported membrane glycoproteins in the axonal tree. J Neurosci 6:1712-1718.

Goldberg DJ, Schacher SE (1987) Differential growth of the branches of a regenerating bifurcate axon is associated with differential axonal transport of organelles. Dev Biol 124:35-40.

Haydon PG, McCobb DP, Kater SB (1984) Serotonin selectively inhibits growth cone motility and synaptogenesis of specific identified neurons. Science 226:561-564.
Innocenti GM (1981) Growth and reshaping of axons in the establishment of visual callosal connections. Science 212:824-827.

Jellies J, Loer CM, Kristan WB Jr (1987) Morphological changes in leech Retzius neurons after target contact during embryogenesis. J Neurosci 7:2618-2629.

LaMantia AS, Rakic P (1990) Axon overproduction and elimination in the corpus callosum of the developing Rhesus monkey. J Neurosci 10:2156-2157.

Land PW, Lund RD (1979) Development of the rat's uncrossed retinotectal pathway and its relation to plasticity studies. Science 205:698-700.

LeVay S, Stryker MP, Shatz CJ (1978) Ocular dominance columns and their development in layer IV of the cat's visual cortex: a quantitative study. J Comp Neurol 179:223-244.

LeVay S, Wiesel TN, Hubel DH (1980) The development of ocular dominance columns in normal and visually deprived monkeys. J Comp Neurol 191:1-51.

Loer CM, Jellies J, Kristan WB (1987) Segment-specific morphogenesis of leech Retzius neurons requires particular peripheral targets. J Neurosci 7:2630-2638.

Loer CM, Kristan WB (1989) Peripheral target choice by homologous neurons during embryogenesis of the medicinal leech. I. Segmentspecific preferences of Retzius cells. J Neurosci 9:513-527.

Luo Y, Raible D, Raper JA (1993) Collapsin: a protein in brain that induces the collapse and paralysis of neuronal growth cones. Cell 75:217-227.

Mattson MP, Dou P, Kater SB (1988) Outgrowth regulating actions of glutamate in isolated hippocampal pyramidal neurons. J Neurosci 8:2087-2100.

McCobb DP, Cohen CS, Connor JA, Kater SB (1988) Interactive effects of serotonin and acetylcholine on neurite elongation. Neuron $1: 377-385$

McLoon SC (1982) Alteration in precision of the crossed retinotectal projection during chick development. Science 215:1418-1420.

Murphey RK, Lemere CA (1984) Competition controls the growth of an identified axonal arborization. Science 224:1352-1355.

O'Leary DDM, Stanfield BB, Cowan WM (1981) Evidence that the early postnatal restriction of the cells of origin of the corpus callosal projections is due to the elimination of axon collaterals rather than to the death of neurons. Dev Brain Res 1:607-617.

O'Leary DDM (1992) Development of connectional diversity and specificity in the mammalian brain by the pruning of collateral projections. Curr Opin Neurobiol 2:70-77.

O'Rourke NA, Cline HT, Fraser SE (1994) Rapid remodeling of retinal arbors in the tectum with and without blockade of synaptic transmission. Neuron 12:921-934.

Redfern PA (1970) Neuromuscular transmission in new-born rats. J Physiol (Lond) 209:701-709.

Schneider G (1973) Early lesions of superior colliculus: factors affecting the formation of abnormal retinal projections. Brain Behav Evol 8:73-109.

Schwab ME, Kapfhammer JP, Bandtlow CE (1993) Inhibitors of neurite growth. Annu Rev Neurosci 16:565-595.

Simoni A, Pellegrini M, Cecconi C, Pellegrino M (1990) Axotomy affects density but not properties of potassium leak channels, in the leech AP neurons. Brain Res 522:118-124.

Smalheiser NR, Crain SM (1984) The possible role of "sibling neurite bias" in the coordination of neurite extension, branching, and survival. J Neurobiol 15:517-529.

Stahl B, Müller B, von Boxberg Y, Cox EC, Bonhoeffer F (1990) Biochemical characterization of a putative axonal guidance molecule of the chick visual system. Neuron 5:735-743.

Stanfield BB, O'Leary DDM (1985) The transient corticospinal projection from the occipital cortex during the postnatal development of the rat. J Com Neurol 238:236-248.

Wallace BG (1984) Selective loss of neurite during differentiation of cells in the leech central nervous system. J Comp Neurol 228:149-153.

Wolszon LR, Gao WQ, Passani MB, Macagno ER (1994a) Growth cone "collapse" in vivo: are inhibitory interactions mediated by gap junctions? J Neurosci 14:999-1010.

Wolszon LR, Rehder V, Kater SB, Macagno ER (1994b) Calcium wave fronts that cross gap junctions may signal neuronal death during development. J Neurosci 14:3437-3448. 